\title{
The AFMC electives diversification policy: Potential drawbacks and benefits for medical students applying to urology
}

\author{
David-Dan Nguyen ${ }^{1}$; Jason Y. Lee ${ }^{2}$; Félix Couture ${ }^{3}$; Patrick O. Richard ${ }^{3}$; Nader Fahmy ${ }^{4}$; Paul \\ Perrotte $^{5}$; Trustin Domes ${ }^{6}$; Naeem Bhojani ${ }^{5}$ \\ ${ }^{1}$ Faculty of Medicine, McGill University, Montreal, QC, Canada; ${ }^{2}$ Division of Urology, University of Toronto, \\ Toronto, ON, Canada; ${ }^{3}$ Division of Urology, Université de Sherbrooke, Sherbrooke, QC, Canada; ${ }^{4}$ Division of \\ Urology, McGill University, Montreal, QC, Canada; ${ }^{5}$ Division of Urology, Université de Montréal, Montreal, QC, \\ Canada; ${ }^{6}$ Division of Urology, University of Saskatchewan, Saskatoon, SK, Canada
}

Cite as: Can Urol Assoc J 2019 April 26; Epub ahead of print. http://dx.doi.org/10.5489/cuaj.6000

Published online April 26, 2019

$* * *$

Clerkship is an important and formative period for future physicians. It involves a combination of core rotations and electives. Core rotations are mandatory and are mainly done at one's home institution, where all students complete the same ones. While the length and content of the core offerings can vary between institutions, accreditation standards ensure that they are similar. Electives, while also mandatory for the completion of the medical curriculum, allow students to personalize the scope of their medical training. They are opportunities for students to do clinical or research rotations in the specialties of their choice and at the locations of their choice, generally prior to making a career decision in the fourth year of medical school.

The increasingly competitive nature of the residency-matching process, as evidenced by the increasing number of unmatched graduates ${ }^{1}$, has led students to invest a majority (if not all) of their available elective weeks in a single specialty with remaining electives strategically used to further strengthen their application for their specialty of interest. This is perceived as demonstrating one's commitment to a specialty and is an opportunity to network with potential residency programs. More importantly, a student's performance during a given elective at a specific institution is consistently cited by Canadian and American residency selection committees as a critical factor in selecting residents. ${ }^{2-4}$ This is also the case for Canadian urology programs. In a survey of Canadian urology selection committees, which will be presented at the 2019 CUA Meeting, performing well during an elective at the responder's school received the highest mean score of importance when selecting new trainees. ${ }^{5}$ In 2019, urology programs gave 175 ranks to Canadian medical graduates (CMGs) that did an elective at their school, and only 52 to those that did not. Of the 28 CMGs that matched last year, all took an elective at their matched school. ${ }^{6}$ While there are always exceptions to the rule, there is undoubtedly increasing 
pressure to decide on a specialty early on in order to secure electives at multiple schools - this is especially true for those eyeing competitive fields such as urology.

Medical educators negatively view students putting all of their eggs in a single basket for two main reasons. First, doing so contradicts the educational intentions of electives, which are to diversify one's exposure and enrich their medical education in order to form well-rounded healthcare providers. Second, such a practice puts an applicant at a higher risk of going unmatched as no serious contingency application to another specialty can be credibly submitted to the Canadian Residency Matching Service (CaRMS). This led the Association of Faculties of Medicine Canada's (AFMC) Undergraduate Medical Education (UGME) Committee to propose the AFMC Student Elective Diversification Policy (SEDP). It reads as the following:

Undergraduate programs recognize their dual responsibility to ensure students undertake a full educational experience that prepares them for any potential career choice, while also optimizing their ability to engage in the increasingly competitive postgraduate match process. Undertaking elective experiences exclusively in a single discipline is pedagogically unsound and fails to provide students with a full exposure to potential career options. Consequently, we commit that, beginning with the Class of 2021, student elective opportunities cannot exceed a maximum of eight weeks in any single entry-level discipline. ${ }^{*}$

By enforcing a national cap on the number of elective weeks spent in an entry-level specialty, the AFMC aims to enhance student learning experiences through more diverse exposure while allowing them to have a contingency strategy with an alternate entry-specialty without seeming less committed. This policy also alleviates the elective application burden for each discipline, thus allowing more candidates who are undecided to secure electives more easily.

How will the adoption of the SEDP affect urology applicants? In 2018, the average number of electives taken by CMGs that matched to urology was 6.7 electives. ${ }^{6}$ Prior to the SEDP, assuming the minimum number of weeks for an elective, which is 2 weeks (and thus potentially underestimating the actual average number of weeks), matched CMGs spent at least 13.4 weeks in urology electives. With the SEDP capping the number of weeks to 8, this represents a decrease in the average number of elective weeks in urology by at least 5.4 weeks per applicant, which could represent a minimum of two to three fewer sites visited.

This decision will result in applicants having fewer direct encounters with urology programs prior to CaRMS. Given the current resident-selection culture, this significantly impacts a candidate's chances of matching to a non-visited program. According to the previously stated

\footnotetext{
* An entry-level discipline is an Entry Route in the PGY-1 (R1) match. Each of these entry-level disciplines leads to specialty certification with either the RCPSC or the CCFP. Electives in subspecialties that are part of a PGY-3 (R3) match (such as the subspecialties in Internal Medicine and Pediatrics) are counted as separate disciplines. As such, electives in these subspecialties do not count towards the 8-week maximum in the general specialty.
} 
evidence, an applicant's performance during an elective at the program's institution directly influences one's chance at matching to that school. The strategy behind elective planning may change when the SEDP is adopted. With a maximum of 8 weeks dedicated to urology, should candidates aim for shorter electives at more schools or longer electives at fewer schools? Will they do more informal site visits to learn about programs they were not able to visit for a clinical elective? What about research electives, which are not considered within an entry-specialty? Will these electives allow students to spend more than 8 weeks in the field of urology?

The effects of increased parallel-planning, particularly in specialties/programs notoriously known for their emphasis on commitment, also remain to be seen. While the authors of this paper believe that parallel planners should not be penalized, how will they compare to applicants with profiles specifically geared towards postgraduate urology training such that urology program selection committees do not have any reservations about which specialty is the applicant's priority (i.e. 8 weeks in urology and use of remaining electives in diverse fields indirectly related to urology such as nephrology, reconstructive surgery, genitourinary medical oncology, etc.)? Indeed, a concern surrounding the SEDP is that certain "strategies" will become the new norm, further limiting the possibility of parallel-planning and exploring unrelated medical fields of interest. A final consideration of increased parallel-planning is the possible increase in the number of applications. As parallel-planning becomes more feasible with the SEDP, one can expect that applicants who were previously undecided between urology and another specialty will now apply to both. While this increase in applicants will certainly allow for a more diverse pool of applicants, how will it affect the ability of post-graduate medical education (PGME) urology programs to evaluate these applications?

By limiting the number of electives in a given discipline, the reduced exposure may put some applicants at a disadvantage not only from the perspective of reduced interviews offered or lower rankings by programs they were unable to visit, but it also reduces their ability to fully evaluate the various programs across the country. As much as applicants are "interviewing” for residency positions, the applicants should also be "interviewing" the programs to determine if they will thrive in that specific learning environment and if their career goals align with the training program's goals. Though all residency programs across Canada provide excellent urologic training, there are slight variations in education style, institutional objectives, faculty phenotype and clinical volume/exposure/practice patterns that result in better or worse fits for different trainees. This reduced exposure to different sites may have specific ramifications for Canadian urology. Since 1994, Canadian urology residency training programs have employed the "Canadian Urology Fair” — a single-site (Toronto, ON for the anglophone universities and Drummondville, QC for the francophone universities), 1-day event to conduct the personal interview portion of the residency selection process. This model made the interview process less expensive and time-consuming for both candidates and faculty. ${ }^{8}$ For many programs, the fair is an opportunity to re-familiarize oneself with a student that has come by one's training center. 
However, with the SEDP, the Canadian Urology Fair further reduces urology applicants’ exposure to different sites in comparison to students applying to other specialties. The Urology Fair might need to change back to the traditional "road show", where students go across Canada to be interviewed allowing them to visit the facilities and spend more time with programs.

While we raise many questions and concerns, the advantages of the SEDP are considerable and undeniable. As previously mentioned, the SEDP ensures that students receive a more balanced education and increased exposure to different specialties prior to entering residency. As outlined by the policy, it will reduce the culture of committing to a single specialty and not parallel plan, hopefully addressing the unmatched issue. Another benefit of the SEDP is attracting a wider range of students to specialties they might not have previously considered such as urology. This ensures that top candidates, regardless of early exposure/interest to urology, can apply to urology. At the very least, this policy will likely increase exposure to urology through electives. A recent pan-Canadian survey of all 17 undergraduate urology education coordinators found that only one Canadian medical school has a mandatory urology clerkship rotation (1 week in duration) while all the other schools offer a selective experience with only approximately $24.3 \%$ (range $5-50 \%$ depending on the school) of Canadian medical students completing this selective experience. Finally, by balancing the playing field and imposing identical limitations on electives to all students, not doing an elective at an institution won't necessarily decrease one's chances to match there. Programs hoping to recruit the best candidates will likely have to rank a certain number of applicants that don't do electives at their institution. The previously cited data highlighting programs' current preference for students that have performed well during a rotation at their school reflects a mentality that will need to change in order to optimize the SEDP.

Only time will tell if the SEDP will achieve its objectives of reducing the unmatched rate while enhancing students' learning experiences. Additionally, there may be unintended consequences that result from the new SEDP, with potential impact on students, programs, and administrative processes alike. While its implications on Canadian UGME and PGME urology education remain to be seen, an adaptation period is unavoidable both for students and selection committees wishing to make the best out of it. For example, direct evaluation of the candidate in the context of an elective will be challenging as students will not be able to visit each institution or will visit for shorter periods of time. This implies that programs may not get the opportunity to fully assess all the top candidates. It is conceivable that traditional assessment methods will need to evolve to assist programs in resident selections-hopefully for the better. 


\section{References}

1. Silverberg SL, Purdy KMH. Unmatched Canadian medical graduates. CMAJ. 2018;190(4):E118. doi:10.1503/cmaj.68737

2. Krauss EM, Bezuhly M, Williams JG. Selecting the best and brightest: A comparison of residency match processes in the United States and Canada. Plast Surg (Oakville, Ont). 2015;23(4):225-230.

3. Eneh AA, Jagan L, Baxter S. Relative importance of the components of the Canadian Residency Matching Service application. Can J Ophthalmol. 2014;49(5):407-413. doi:10.1016/j.jcjo.2014.06.009

4. Weissbart SJ, Stock JA, Wein AJ. Program directors' criteria for selection into urology residency. Urology. 2015;85(4):731-736. doi:10.1016/j.urology.2014.12.041

5. Nguyen D-D, Lee JY, Valdivieso R, Franc-Guimond J, Bhojani N. Survey of Canadian urology programs: Which elements of the CaRMS application are the most important? In: CUA. ; 2019.

6. Canadian Residency Matching Service. https://www.carms.ca/. Accessed March 25, 2019.

7. The Association of Faculties of Medicine Canada. AFMC Student Electives Diversification Policy | The Association of Faculties of Medicine of Canada - Today's Research, Tomorrow's Doctors. https://afmc.ca/news/2019-03-25. Published 2019. Accessed March 25, 2019.

8. Grober ED, Matsumoto ED, Jewett MAS, Chin JL, Canadian Urology Program Directors and the CUP. The Canadian Urology Fair: a model for minimizing the financial and academic costs of the residency selection process. Can J Surg. 2003;46(6):458-462. 